\title{
Methotrexate-Associated Lymphoproliferative Disorder: Dermoscopic Features
}

\author{
Takeshi Namiki $^{a} \quad$ Yumiko Sone $^{a} \quad$ Keiko Miura $^{b} \quad$ Masaru Tanaka $^{c}$ \\ Hiroo Yokozeki ${ }^{a}$
}

aDepartment of Dermatology, Graduate School of Medical and Dental Sciences, Tokyo Medical and Dental University, Tokyo, Japan; ${ }^{b}$ Department of Pathology, Graduate School of Medical and Dental Sciences, Tokyo Medical and Dental University, Tokyo, Japan; 'Department of Dermatology, Tokyo Women's Medical University Medical Center East, Tokyo, Japan

\section{Keywords}

Methotrexate $\cdot$ Lymphoproliferative disorder $\cdot$ Dermoscopy $\cdot$ Epstein-Barr virus

\begin{abstract}
Methotrexate-related lymphoproliferative disorder (MTX-LPD) is a rare disorder caused by long-term MTX therapy for autoimmune diseases. There has been no report of the dermoscopic features of MTX-LPD to date. A 64-year-old female presented with a slightly elevated indurated erythematous plaque with scales on her right thigh. The patient had been treated for rheumatic arthritis with MTX and prednisolone for more than 15 years, and $18 \mathrm{mg} /$ week MTX without prednisolone had been administered in the last year. Dermoscopy revealed dotted vessels and glomerular vessels on pink homogeneous areas and multiple surface scales. Enhanced computed tomography showed multiple nodules and lymphadenopathies at the mediastinum and axillae. Histopathological examination revealed telangiectasia in the superficial dermis. Atypical lymphoid cells were scattered in the whole dermis and subcutaneous tissue. A perivascular infiltrate of atypical lymphocytes and histiocytoid cells partially destroyed the vessel walls. Epstein-Barr virus in situ hybridization showed a positive result. The cessation of MTX reduced the erythematous plaque, and lymphadenopathies at the neck, mediastinum,
\end{abstract}


and axillae were not palpable. We discuss the relevance of these dermoscopic and histopathological features. The accumulation of such cases will reveal the dermoscopic features of MTXLPD and the utility of dermoscopy for the diagnosis of MTX-LPD.

(c) 2018 The Author(s)

Published by S. Karger AG, Basel

\section{Introduction}

Methotrexate-related lymphoproliferative disorder (MTX-LPD) is a rare disorder caused by long-term MTX therapy for autoimmune diseases [1,2]. Although a careful examination of skin lesions is critical for an accurate diagnosis of MTX-LPD, no report of dermoscopic features has been published. Here, we report a case of MTX-LPD and, for the first time, present its dermoscopic features.

\section{Case Report}

A 64-year-old female presented with an erythematous plaque on her right thigh. The patient had received prednisolone, JAK inhibitor, certolizumab, and infliximab $8 \mathrm{mg} / \mathrm{kg}$ for rheumatoid arthritis over a span of 15 years and had started $18 \mathrm{mg} /$ week of MTX 4 years before presentation, which persisted with the infliximab at presentation. A subcutaneous, tender, erythematous tumor had appeared on her right thigh 1 month after the cessation of prednisolone. The patient consulted the Department of Dermatology due to no remission 1 month after the cessation of infliximab. Physical examination revealed a slightly elevated indurated erythematous plaque with scales on her right thigh (Fig. 1a). Dermoscopy revealed ambiguous dotted/glomerular vessels on pink homogeneous areas (Fig. 1b), which were clearly visible with contrast enhancement (Fig. 1c), and multiple surface scales (Fig. 1b, c). Enhanced computed tomography showed multiple nodules and lymphadenopathies at the mediastinum and axillae. A bone marrow biopsy revealed a negative result. Histopathological examination revealed perivascular infiltrates of atypical lymphocytes and histiocytoid cells in the dermis and subcutaneous tissues (Fig. 2a, b). Atypical lymphocytes with halos infiltrated the small vessels (Fig. 2c). Partial destruction of vessels compensatively expanded vessels in the superficial dermis (Fig. 2d). Immunohistochemistry showed positive reactions for CD15, CD20 (Fig. 2e), CD30, CD79a, and Bcl-2 and negative reactions for CD3, CD5, CD7, CD10, CD56, granzyme B, TIA-1, and ALK. Positivity of atypical lymphoid cells was $80 \%$. Epstein-Barr virus-encoded RNA in situ hybridization showed a positive result (Fig. 2f). Immunohistochemistry for CD34 and $\alpha$-SMA highlighted dilated vessels in the superficial dermis (Fig. 2g). Both histopathological and immunohistochemical findings did not imply Epstein-Barr virus-positive Hodgkin lymphoma. The erythematous plaques disappeared 1 month after the cessation of MTX. The diagnosis of MTX-LPD was made. Lymphadenopathies at the neck, mediastinum, and axillae were reduced 4 months after the cessation of MTX.

\section{Discussion}

MTX-LPD is an LPD caused by MTX and is categorized under iatrogenic immunodeficiency-associated LPD. Cutaneous manifestations of MTX-LPD are nodules and/or erythematous plaques [3]. Although vascular findings of dermoscopy are difficult to be specified in the 
differential diagnosis between amelanotic melanomas, nonpigmented Bowen disease, and other cutaneous lymphomas, the lack of both apparent polymorphous vessels and dermoscopic findings of the epidermal structures may not imply the diagnosis of amelanotic melanomas and nonpigmented Bowen disease. In cutaneous lymphomas, serpentine vessels and fine short linear vessels are frequent in primary B-cell lymphoma and mycosis fungoides, respectively $[4,5]$. In our case, dotted/glomerular vessels on pinkish backgrounds are the main dermoscopic features. Those dermoscopic features of vascular structures may partially reflect histopathological features of vascular damages by atypical lymphocytes. Although it is difficult to specify a particular subset of cutaneous lymphomas by dermoscopy, accumulation of such cases may reveal frequent dermoscopic features of MTX-LPD.

\section{Statement of Ethics}

Informed consent has been obtained and no ethics approval was required for reporting of a single case.

\section{Disclosure Statement}

The authors have no conflicts of interest to declare.

\section{References}

1 Kamel OW, van de Rijn M, Weiss LM, Del Zoppo GJ, Hench PK, Robbins BA et al. Brief report: reversible lymphomas associated with Epstein-Barr virus occurring during methotrexate therapy for rheumatoid arthritis and dermatomyositis. N Engl J Med. 1993 May;328(18):1317-21.

2 Shimura C, Satoh T, Takayama K, Yokozeki H. Methotrexate-related lymphoproliferative disorder with extensive vascular involvement in a patient with rheumatoid arthritis. J Am Acad Dermatol. 2009 Jul;61(1):126-9.

3 Shimizu S, Inokuma D, Murata J, Kikuchi K, Ito T, Fukasawa Y et al. Cutaneous manifestations of methotrexate-associated lymphoproliferative disorders: report of two cases and a review of the literature. Acta Derm Venereol. 2015 Mar;95(3):366-7.

4 Lallas A, Apalla Z, Lefaki I, Tzellos T, Karatolias A, Sotiriou E et al. Dermoscopy of early stage mycosis fungoides. J Eur Acad Dermatol Venereol. 2013 May;27(5):617-21.

5 Geller S, Marghoob AA, Scope A, Braun RP, Myskowski PL. Dermoscopy and the diagnosis of primary cutaneous B-cell lymphoma. J Eur Acad Dermatol Venereol. 2018 Jan;32(1):53-6. 


\section{Case Reports in Dermatology}
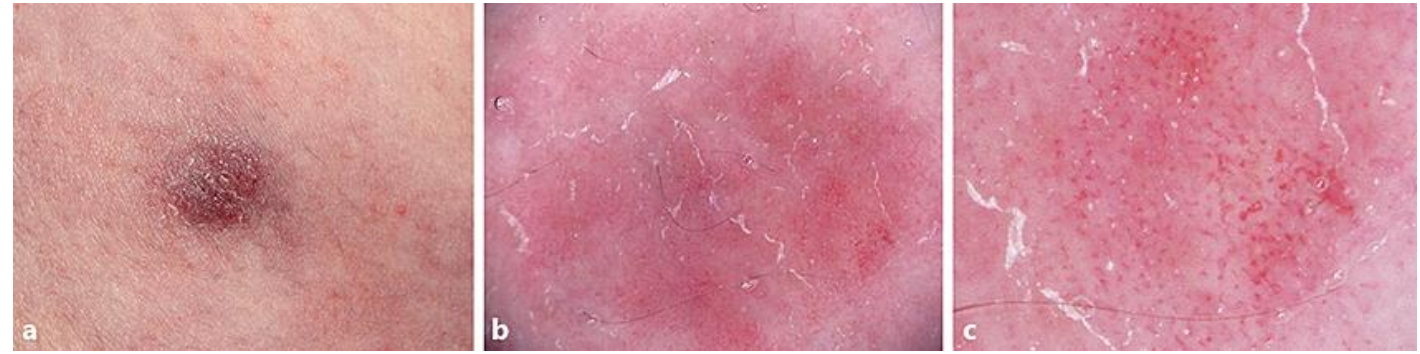

Fig. 1. a Slightly elevated indurated erythematous plaque with scales on the patient's right thigh. b Dermoscopy showing multiple surface scales and pink homogeneous areas. c Contrast-enhanced image of the dermoscopic features; dotted/glomerular vessels were noted at the center of the lesion. 


\section{Case Reports in Dermatology}

\begin{tabular}{l|l}
\hline Case Rep Dermatol 2018;10:149-153 \\
\hline DOI: 10.1159/000489694 & $\begin{array}{l}\text { ○ 2018 The Author(s). Published by S. Karger AG, Basel } \\
\text { www.karger.com/cde }\end{array}$ \\
\hline
\end{tabular}

Namiki et al.: Methotrexate-Associated Lymphoproliferative Disorder: Dermoscopic Features
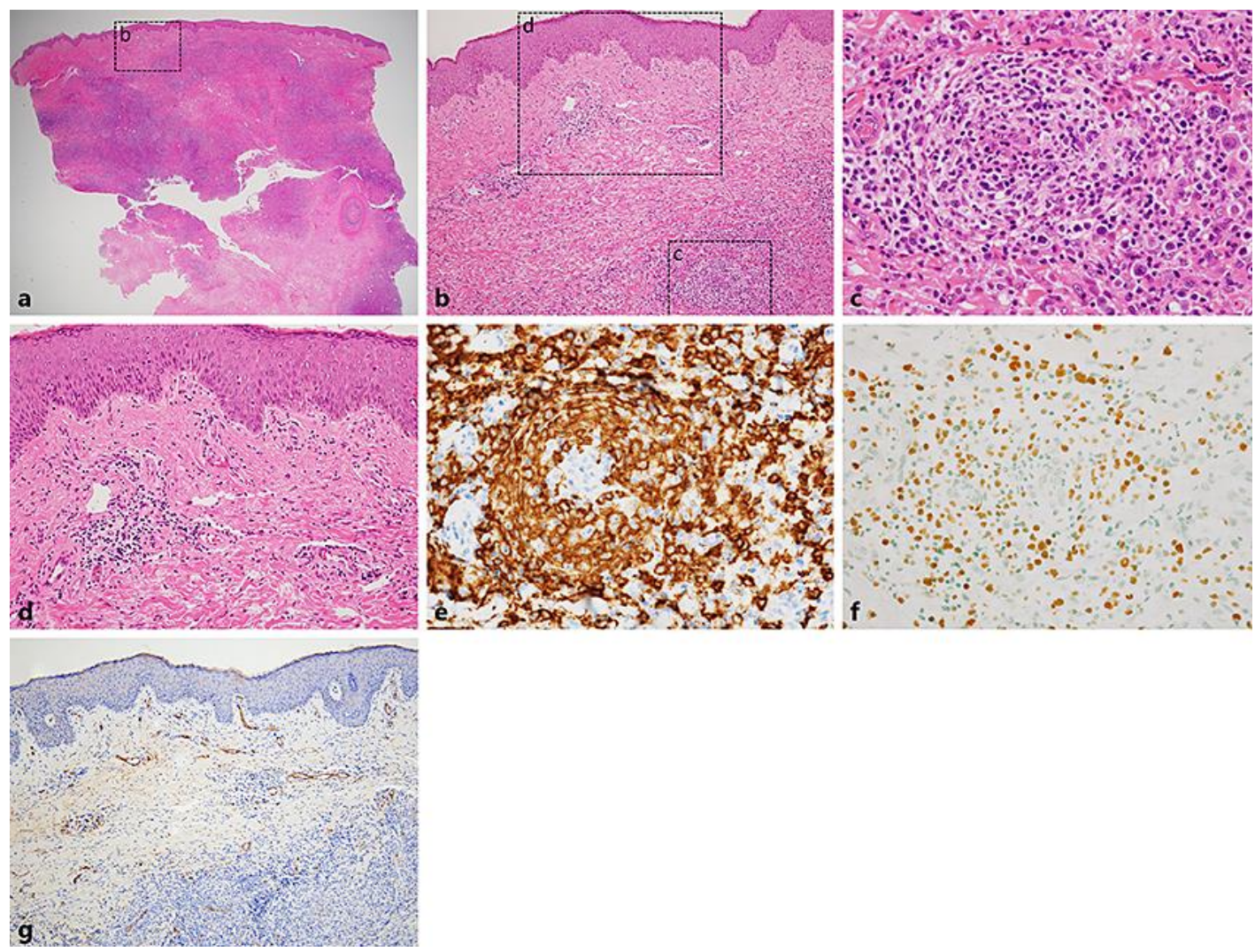

Fig. 2. a Histopathology showing perivascular infiltrates of lymphoid cells in the dermis and subcutaneous tissues; the dotted box labelled "b" indicates an area shown in b (HE stain; original magnification, $\times 12.5$ ). b Dilated vessels were found in the superficial dermis, and a perivascular infiltrate of atypical lymphocytes and histiocytoid cells partially destroyed the vessel walls; dotted boxes labelled "c" and "d" indicate areas shown in $\mathbf{c}$ and $\mathbf{d}$, respectively (HE stain; original magnification, $\times 100$ ). c Atypical lymphocytes with halos infiltrated the vessels; some atypical lymphocytes with halos had convoluted and enlarged nuclei (HE stain; original magnification, $\times 400$ ). $\mathbf{d}$ Partial destruction of vessels compensatively expanded vessels in the superficial dermis (HE stain; original magnification, $\times 200$ ). e Immunohistochemistry for CD20 showing a positive reaction in atypical lymphocytes; the area shown is the same as in c (original magnification, $\times 400$ ). $f$ Epstein-Barr virus-encoded RNA in situ hybridization and a positive result in atypical lymphocytes; the area shown is the same as in e (original magnification, $\times 400$ ). $\mathbf{g}$ Immunohistochemistry for CD34 revealing dilated vessels in the superficial dermis (original magnification, $\times 100$ ). 